\title{
Assessment of Volatile Compound Profiles and the Deduced Sensory Significance of Virgin Olive Oils from the Progeny of Picual x Arbequina Cultivars
}

Ana G. Pérez ${ }^{1}$, Raúl de la Rosa ${ }^{2}$, Mar Pascual ${ }^{1}$, Araceli Sánchez-Ortiz ${ }^{1}$, Carmen RomeroSegura $^{1}$, Lorenzo León ${ }^{2}$, Carlos Sanz ${ }^{1}$

${ }^{1}$ Department of Biochemistry and Molecular Biology of Plant Products, Instituto de la Grasa, CSIC, Ctra. Utrera km 1, Campus University Pablo de Olavide, Building 46, 41013-Seville, Spain.

${ }^{2}$ IFAPA, Centro Alameda del Obispo, Menéndez Pidal s/n, 14004-Córdoba, Spain.

Corresponding author: Carlos Sanz, Department of Biochemistry and Molecular Biology of Plant Products, Instituto de la Grasa, CSIC, Ctra. Utrera km 1, Campus University Pablo de Olavide, Building 46, 41013-Seville, Spain. Tel: +34 954611550, Fax: +34 954616790; email: carlos.sanz@ig.csic.es 
2

3 Volatile compounds are responsible for most of the sensory qualities of virgin olive oil and

\section{Abstract}

they are synthesized when enzymes and substrates come together as olive fruit is crushed during the industrial process to obtain the oil. Here we have studied the variability among the major volatile compounds in virgin olive oil prepared from the progeny of a cross of Picual and Arbequina olive cultivars (Olea europaea L.). The volatile compounds were isolated by SPME, and analyzed by HRGC-MS and HRGC-FID. Most of the volatile compounds found in the progeny's oil are produced by the enzymes in the so-called lipoxygenase pathway, and they may be clustered into different groups according to their chain length and polyunsaturated fatty acid origin (linoleic and linolenic acids). In addition, a group of compounds derived from amino acid metabolism and two terpenes also contributed significantly to the volatile fraction, some of which had significant odor values in most of the genotypes evaluated. The volatile compound content of the progeny was very varied, widely transgressing the progenitor levels, suggesting that in breeding programs it might be more effective to consider a larger number of individuals within the same cross than using different crosses with fewer individuals. Multivariate analysis allowed genotypes with particularly interesting volatile compositions to be identified and their flavor quality deduced.

Keywords: Olea europaea L., virgin olive oil, volatile compounds, variability, segregation, quality 5 


\section{Introduction}

Olive oil is one of the oldest known plant oils and it is unique as it can be consumed as a fruit juice called virgin olive oil (VOO). This product represents the primary source of lipids in the Mediterranean diet, which has been linked to positive health benefits. Indeed, this diet reduces the risk from a number of diseases, mainly those containing an inflammatory component such as cardiovascular diseases, certain types of cancer, diabetes, metabolic syndrome, arthritis and Alzheimer's disease [1-6]. Thus, increased VOO consumption, one of the main distinguishing features of the Mediterranean diet, is likely to have a positive impact on the general population's health and consequently, on the budgets allocated to healthcare systems. However, the increase in the demand for high-quality VOO may not only be attributed to its potential health benefits but also, to its excellent organoleptic properties [7]. In this sense, volatile compounds are responsible for the aroma of VOO, which is characterized by a unique balance of green and fruity attributes spiced with some other positive aromas that make it a distinctive edible oil. The size, shape, conformation, type and position of the functional groups in volatile compounds are features that strongly influence odor perception and pleasantness $[8,9]$. On the other hand, concentration and odor threshold of each volatile compound define its sensory attributes. These parameters determine the odor activity value (OAV), the ratio between the concentration of the volatile compound and its odor threshold, whereby volatile compounds with an OAV below one do not contribute to VOO aroma. An interesting approach to understand the relationship between volatile compounds and odor attributes is the statistical sensory wheel (SSW) developed by Aparicio and Morales [10], which compiles the sensory attributes evaluated by trained VOO sensory panels across Europe. The resulting information allows sensory notes with a similar semantic description to be clustered into a number of sectors that contain the volatile compounds generally identified by a given sensory perception, and that among the most relevant to VOO aroma may include green or ripe fruit odor notes.

Most of the volatile compounds present in VOO are synthesized when the enzymes and substrates come together when the olive fruit is crushed during the industrial process to obtain this product. The lipoxygenase (LOX) pathway participates in the biosynthesis of six straightchain carbons (C6) compounds in the volatile fraction of VOO [11] and from a quantitative or qualitative point of view, C6 aldehydes and alcohols, as well as their corresponding esters, are the most important compounds in VOO aroma $[12,13]$. These compounds are synthesized 
from polyunsaturated fatty acids containing a $(Z, Z)-1,4$-pentadiene structure, such as linoleic (LA) and linolenic (LnA) acids. In the first step of this pathway, LOX produces the corresponding 13-hydroperoxide derivatives that are subsequently cleaved heterolytically by hydroperoxide lyase (HPL) to C6 aldehydes [11, 14, 15]. C6 aldehydes can then by reduced by alcohol dehydrogenases (ADHs) to C6 alcohols $[11,16]$ and finally, they can be transformed into the corresponding esters by alcohol acyltransferases (AATs) $[11,17]$. Compounds with five straight-chain carbons (C5 compounds) are also relevant to the aroma of olive oil [13] and they are generated through an additional branch of the LOX pathway that involves the production of a 13-alcoxyl radical by LOX, as demonstrated in soybean seeds [18]. This radical can undergo subsequent homolytic non-enzymatic $\beta$-scission to form a 1,3pentene allylic radical that can be chemically dimerized to generate pentene dimers (PD), or that reacts with a hydroxyl radical to form C5 alcohols. The latter represents the origin of the C5 carbonyl compounds present in the volatile fraction of olive oil through enzymatic oxidation by $\mathrm{ADH}$, as believed to occur in soybean leaves [19].

New cultivars with improved sensory quality might further stimulate VOO consumption and although olive breeding programs have traditionally focused mainly on the improvement of agronomic traits [20], more recent breeding studies have addressed selection for the sensory and nutritional qualities of VOO [21, 22]. Considering the significance of the aroma to VOO quality, the aim of the present work was to assess the variation in the volatile fraction of VOO and to deduce the aroma properties in a segregating population of a cross of Picual $\mathrm{x}$ Arbequina olive cultivars. This study was carried out in the framework of an olive breeding program that aimed to identify new olive genotypes that give rise to oils with improved sensory and nutritional attributes.

\section{Materials and Methods}

\subsection{Plant material}

A total of 136 olive genotypes (Olea europaea L.) from a Picual x Arbequina cross were considered in the present study. The two parental cultivars, Picual and Arbequina, were grown in the same orchard as the seedling progeny. The cross was made in spring 2001 and the seedlings obtained were submitted to the habitual protocol followed on the breeding program [23]. Initial seedling growth was forced in a greenhouse by means of drip fertirrigation, 
temperature control and continuous light. The plants were then established in open field in September 2003 with a spacing of $1.5 \times 4 \mathrm{~m}$, trained to form a canopy at $160 \mathrm{~cm}$ height and then allowed to develop freely. Drip irrigation and standard culture practices were followed to ensure tree growth without limitations. All trees were grown in the same edaphoclimatic conditions at the experimental orchards of IFAPA (Alameda del Obispo, Córdoba, Spain). Fruit was picked by hand on three consecutive years (2008-2010) when it reached an average ripening index of 2.5 (turning stage) to better compare the genotypes (according to El Riachy et al. [24]).

\subsection{Olive oil extraction}

Olive oil was extracted using an Abencor analyzer (Comercial Abengoa, S.A., Seville, Spain) that simulates the industrial process of VOO production on a laboratory scale [25]. Milling of the olive fruit was performed using a stainless steel hammer mill operating at $3000 \mathrm{rpm}$ and with a $5 \mathrm{~mm}$ sieve. Malaxation was carried out for $30 \mathrm{~min}$ with the Abencor thermobeater operated at $30{ }^{\circ} \mathrm{C}$ according to industrial recommendations. Centrifugation of the kneaded paste was performed in a basket centrifuge at $3500 \mathrm{rpm}$ for $1 \mathrm{~min}$ and after centrifugation, the oils were decanted and paper filtered. Oils were stored under nitrogen at $-20{ }^{\circ} \mathrm{C}$ until they were analyzed.

\subsection{Analysis of volatile compounds}

Olive oil samples were conditioned to room temperature and then placed in a vial heater at 40 ${ }^{\circ} \mathrm{C}$. After a 10 min equilibration, volatile compounds from the headspace were adsorbed onto SPME fiber DVB/Carboxen/PDMS 50/30 $\mu \mathrm{m}$ (Supelco Co., Bellefonte, PA). The sampling time was $50 \mathrm{~min}$ at $40{ }^{\circ} \mathrm{C}$ and desorption of volatile compounds trapped in the SPME fiber was performed directly into the GC injector. Volatile compounds were identified out on a 7820A/GC-5975/MSD system (Agilent Technologies), equipped with a DB-Wax capillary column $(60 \mathrm{~m} \times 0.25 \mathrm{~mm}$ i.d., film thickness, $0.25 \mu \mathrm{m}$ : J\&W Scientific, Folsom, CA $)$ and under the following conditions: the injection port was operated in splitless mode at $250{ }^{\circ} \mathrm{C}$; He was used as the carrier gas and the flow rate was $1 \mathrm{~mL} / \mathrm{min}$; column was held for $6 \mathrm{~min}$ at $40{ }^{\circ} \mathrm{C}$ and then ramped up at $2{ }^{\circ} \mathrm{C} \min ^{-1}$ to $168^{\circ} \mathrm{C}$; the mass detector was operated in the electronic impact mode at $70 \mathrm{eV}$, the source temperature was set at $230{ }^{\circ} \mathrm{C}$ and the mass 
spectra were scanned at $2.86 \mathrm{scans} / \mathrm{s}$ in the $\mathrm{m} / \mathrm{z} 40-550 \mathrm{amu}$ range (see a sample in Figure $\mathrm{S} 1$ and Table S1 in Supporting Information). The compounds were matched to the Wiley/NBS and NIST libraries and against the GC retention time of available standards. VOO volatile compounds were analyzed three times on a HP-6890 gas chromatography apparatus (Agilent Technologies), which was equipped with a similar column and operated under the following operating conditions in order to obtain quite similar retention times for volatile compounds such as those obtained with the 7820A/GC-5975/MSD system: N2 as the carrier gas at a constant pressure of $17 \mathrm{psi}$; injector and detector at $250{ }^{\circ} \mathrm{C}$; column held for 6 min at $40{ }^{\circ} \mathrm{C}$ and then programmed at $2{ }^{\circ} \mathrm{C} \min ^{-1}$ to $168^{\circ} \mathrm{C}$. Individual calibration curves for each compound were used for quantification by adding known amounts of the different compounds to redeodorized high-oleic sunflower oil.

The volatile compounds were clustered into different groups and subgroups according to the polyunsaturated fatty acid and the origin of the LOX pathway branch, and as the terpene and branched-chain (BC) volatile compounds from amino acid metabolism (Table 1).

\subsection{Statistical analysis}

The data were evaluated using STATISTICA (Statsoft Inc., Tulsa, OK, USA). Correlations among volatile compounds or groups of volatile compounds were analyzed using Pearson's correlation coefficients. Principal component (PCA) and cluster analysis were used to evaluate the associations among the volatile compounds from the progeny.

\section{Results and Discussion}

Taking into account the proven relationship between volatile compounds and the sensorial quality of VOO [7], the volatile fraction of the oils produced from the segregating progeny of a Picual x Arbequina cross was assessed over three consecutive years. These data have now been deposited at the Oleagen web page (https://chirimoyo.ac.uma.es/oleagen). In terms of the content of volatile compounds, the progeny displayed a high degree of variability between individuals, widely transgressing the progenitor levels (Figure 1 and Table 1). A wide variability in such progeny has been reported previously for fruit traits [26] and for other oil components [27]. However, as far as we know there have been no previous reports on the 
segregation of the volatile compound content in VOO. As mentioned in the Introduction, most of the volatile compounds found in the oils of the progeny are produced by the enzymatic systems including in the so-called LOX pathway [11], and they may be clustered into different groups according to chain length and the polyunsaturated fatty acid (C6/LnA, C6/LA, C5/LnA, C5/LA), and the origin of the esters (LOX esters). Previous findings suggest that the synthesis of these compounds depends on the availability of substrates to be catabolized through the LOX pathway during the process of obtaining the oil. Moreover, LOX activity is an important limiting factor for the synthesis of these volatile compounds, although such limitations do not seem to occur in the same direction among the different olive cultivars $[28,29]$. The differences observed in the limitation of the HPL reaction among the olive cultivars seem to be more closely related to the variation in the amount of hydroperoxides synthesized during the oil extraction process of each cultivar than to the HPL activity during olive processing [30]. In addition, a group of compounds that featured a branched-chain (BC) chemical structure derived from amino acid metabolism and two terpenes also contributed significantly to the volatile fraction in the oils isolated from the progeny.

When VOO was obtained from non-sound fruits, such as infested olives or olives collected from the ground, or if VOO was inadequately processed or stored, the profile of volatile compounds in VOO may include compounds that are responsible for off-flavors, predominantly carboxylic acids or aliphatic C8-C11 carbonyls and alcohols [31, 32]. The presence of such compounds is commonly associated with sensory defects in the oils and they are mainly produced by chemical oxidation or through the activity of exogenous enzymes, usually due to microbial activity present in non-sound fruits. In this study, only hand-picked, sound fruit was used in the turning stage, and mild operation conditions were employed to avoid the synthesis of such compounds that cause defects in the oils.

With very few exceptions, most of the volatile compounds in the oils of the progeny were six straight-chain carbon compounds derived from linolenic acid $(\mathrm{C} 6 / \mathrm{LnA})$, the content of which was 2-160 times higher on average than that of the rest of the groups of volatile compounds in the oils (Fig. 1). The C6/LnA compounds varied from 0.50 to $51.99 \mu \mathrm{g} / \mathrm{g}$ oil, a variability that greatly exceeded that found for oils produced from other olive cultivars. The accumulation of C6 compounds studied in 39 monovarietal VOOs obtained from trees cultivated in the same orchard under the same pedoclimatic conditions was in the range of 2.52-18.11 $\mu \mathrm{g} / \mathrm{g}$ oil [33]. Among the C6/LnA compounds, the aldehyde group (C6/LnA 
aldehydes) was the most abundant, $(E)$-hex-2-enal being the main contributor ( $88 \%$ of total C6/LnA compounds on average: Figure 2). The mean content of this compound in the oils was $14.59 \mu \mathrm{g} / \mathrm{g}$ oil, ranging from $0.35-43.38 \mu \mathrm{g} / \mathrm{g}$ oil. These large amounts of $(E)$-hex-2-enal and the relatively low odor threshold (Table 1) mean that this C6/LnA compound is likely to be one of the main contributors to the aroma of the olive oils produced from the progeny. Indeed, this seems to be a common feature of the oils obtained from different olive cultivars [33].

Only the C5/LnA group displayed comparable contents to those of the C6/LnA group of compounds when the pentene dimers were included among them. Pentene dimers are thought to be synthesized during the oil extraction process through the same branch of the LOX pathway as the C5 compounds [34]. A strong relationship between the pentene dimer content and that of the rest of C5/LnA compounds has been observed in this study, which will be discussed later. Pentene dimers represented on average $86 \%$ of the $\mathrm{C} 5 / \mathrm{LnA}$ content in the progeny oils (Fig. 2), although the sensory contribution of pentene dimers to the VOO aroma seems to be quite low or negligible given the estimated odor thresholds for these compounds. While no odor thresholds have been reported for pentene dimers, estimates can be made from the average published values for the structurally related C6-C10 dienes [35], which on average represent 13,500 ng/g oil. Although displaying comparatively lower contents than pentene dimers, the rest of C5/LnA compounds seem to have a notable involvement in the VOO aroma according to their OAVs. Among them, pent-1-en-3-one and the pent-2-en-1-ols are especially noteworthy, in close agreement with earlier findings [36], and all the genotypes displayed pent-1-en-3-one contents above its odor threshold ( $0.73 \mathrm{ng} / \mathrm{g}$ oil: Table 1$)$. The aroma of this compound is described as green pungent and it is considered to provide an unpleasant sensation [37]. However, the aroma of pent-2-en-1-ol is described as green fruity, the typical basic perception of virgin olive oils, reminiscent of healthy, fresh olive fruits harvested at the right ripening stage. Most of the genotypes of the progeny have $(Z)$-pent-2en-1-ol contents below its threshold concentration, suggesting this component is generally of little relevance in terms of contributing to VOO aroma. Conversely, $63 \%$ of the oils from the genotypes had a (E)-pent-2-en-1-ol content above its estimated odor threshold.

On average, terpenes were the third major group of volatile compounds in the oils of the progeny of Picual x Arbequina cross, although their levels were very variable (8-19,653 ng/g oil) compared to those of C5/LnA compounds. In general, limonene seems not to be an important contributor to VOO aroma since only four of the genotypes had an OAV above 1 
for this terpene (Table 1). However, around $65 \%$ of the progeny seemed to have significant levels of ocimene, suggesting that this compound might make an important contribution to the aroma of the oils $(\mathrm{OAV}>1)$ should the odor threshold of ocimene be similar to that of the structurally cycled isomer limonene ( $250 \mathrm{ng} / \mathrm{g}$ oil). As far as we know, no thresholds for ocimene in oil have been reported. Nevertheless, the fact that non-cyclic terpenes generally display lower odor thresholds than their cyclic counterparts suggests that the contribution of ocimene to VOO aroma could be even more prominent.

As mentioned previously, esters convey the fruity odor notes to the oils that are much appreciated by consumers, especially LOX esters. The LOX ester content in the progeny was $871 \mathrm{ng} / \mathrm{g}$ oil on average and in a range of 8-7438 ng/g oil. Only a few genotypes had a hexyl acetate and $(E)$-hex-2-en-1-yl acetate contents consistent with them contributing to VOO aroma (OAV > 1: Table 1), although (Z)-hex-3-en-1-yl acetate did seem to be an important contributor to VOO aroma in around $67 \%$ of the progeny.

The levels of the $\mathrm{BC}$ compounds in the different genotypes of the progeny are also noteworthy. Although these compounds are found at low concentrations in the oils (averaging overall around $100 \mathrm{ng} / \mathrm{g}$ oil and in the range of 15-901 ng/g oil), they could still have a profound impact on the aroma of the oils. The 2-methyl-butan-1-ol content seems to be related to the fusty defects of VOO aroma [38], yet this BC compound was generally found below its odor threshold. Conversely, the 2 and 3-methyl-butanal content suggests that these $\mathrm{BC}$ aldehydes did contribute to the VOO aroma, since in all the genotypes of the progeny the OAV for both these BC aldehydes was $>1$. Indeed, both BC aldehydes were located in the ripe fruit sector of the SSW [10].

When the relationships among the different of groups of volatile compounds in the oils from the Picual x Arbequina cross progeny were studied, the main classes of volatile compounds (C6, C5 and terpenes) were significantly correlated with the total content of volatile compounds in the oils (Table 2). Of particular note was the strong and very significant correlation between the $\mathrm{C} 6 / \mathrm{LnA}$ aldehydes $(r=0.91)$ and the total content of volatile compounds in the oils, as well as the moderate correlation of the C5/LnA carbonyls ( $r$ $=0.49)$ and the pentene dimers $(r=0.70)$ with this parameter. By contrast, BC compounds and esters display a weak negative correlation (BC aldehydes) or almost no correlation with the total volatile compound content of the oils. This is noteworthy given that these compounds may have a strong impact on the aroma of VOO, especially the LOX esters and $\mathrm{BC}$ aldehydes. Moreover, these groups of compounds were not strongly correlated with any 
other group of compounds. While this is reasonable for $\mathrm{BC}$ compounds given that they do not share any synthetic metabolic pathway, it was unexpected for the LOX esters. These esters were weakly correlated with C6/LA alcohol $(r=0.33)$ but not with their main C6/LnA precursor alcohols $(r=0.02)$, which suggests that the activity of the alcohol acyltransferase (AAT) is limited to a large extent among the individual progeny. In fact, we previously found AAT activity to be limited in both progenitors of this progeny, albeit more so in Picual than in Arbequina fruit [39]. It was also concluded that the origin of the low volatile esters arising from the LOX pathway in oils of Arbequina and Picual cultivars was largely due to a limitation on alcohol synthesis during VOO production than to dampened AAT activity. In this sense, there was a weak correlation between the content of the C6/LnA alcohols and their metabolic precursors, the C6/LnA aldehydes ( $r=0.29$ : Table 2$)$, as also observed for the saturated fraction of C6/LA alcohol and C6/LA aldehyde $(r=0.42)$. These data are in accordance with the strong limitation on ADH activity found in the Picual and Arbequina progenitors during oil extraction [39].

Significant correlations were also found between the pentene dimers content and that of C5/LnA carbonyls and alcohols ( $r=0.71$ and 0.61 , respectively). This was similar to that of the latter groups of compounds when compared with each other $(r=0.76)$, suggesting a strong metabolic relationship between these groups of compounds. Indeed, these data support the hypothesis that pentene dimer synthesis during oil extraction process occurs in the same way as C5 alcohols are synthesized, involving the formation of an alkoxy radical from a polyunsaturated fatty acid following the activity of a LOX protein [34]. The fact that the pentene dimer content was not correlated with the C5/LA carbonyl and alcohol content $(r=-$ 0.04 and -0.15 , respectively) suggests that pentene dimers were only produced from LnA and not from LA.

Most of this variability among the VOO volatile profiles seems to correspond exclusively to the genotype, since the genotypes could not be grouped in terms of harvest year when analyzed by a PCA using either all the volatile compounds as the variables or only those that are most important from a sensorial point of view (Figure S2 in Supporting Information). It should be noted that the progeny and progenitors were all grown in the same orchard, under the same edaphoclimatic conditions, and that the oils were extracted in exactly the same way, with no a priori criterion to select the genotypes tested on each of the three sampling years. Indeed, similar results were found when analyzing the phenolic profiles of these progeny oils 
[27], although it has been possible to detect the differentiation of distinct groups when a reduced number of genotypes from an olive cross were compared over consecutive years [40].

A PCA was performed to explain the correlations among the different volatile compounds assessed in the oils of the progeny of the Picual x Arbequina cross (Figure 3). The first two PCs carried a moderate amount of important information, with the first factor explaining $25.73 \%$ of the variance whereas the second factor explained $11.47 \%$. We previously found quite similar values when assessing the content of the main phenolic compounds in the oils of this progeny ( $24.94 \%$ and $16.46 \%$ for factor 1 and 2, respectively) [27]. Most of the C6 and C5 compounds could be grouped separately from the other compounds ( Figure 3A) and especially, the C6 aldehydes and the C5 compounds derived from LnA that cover a region between the second and third quadrants whose variances are basically explained by factor 2 . The only exception is the position of $(Z)$-pent-2-en-1-ol (5C$5)$, clearly distanced from its isomer (E)-pent-2-en-1-ol (5C-6), which suggests a different origin: (E)-pent-2-en-1-ol would be synthesized through the homolytic branch of the LOX pathway, whereas the origin of its $(Z)$-isomer could be more closely related to chemical oxidation. However, this is not consistent with the location in the plot of their theoretical metabolic products, $(Z)$ and $(E)$-pent-2-enal (5C-2 and 5C-3, respectively). The C6 aldehyde (hexanal, 6C-8) and the C5 compounds derived from LA (5C-14, 5C-15 and 5C-16) were also clearly separated from the main group of compounds derived from the LOX pathway (C6 aldehydes and the C5 compounds derived from LnA). Again and although at least partially synthesized through the LOX pathway, the origin of these compounds could be more closely related to pure chemical oxidation processes. Indeed, there may be two different modes of hexanal formation during VOO extraction, through enzymatic and non-enzymatic pathways [41]. The latter may be boosted by the use of high temperatures and longer times during the malaxation of the olive paste.

C6 alcohols from both LA and LnA could be also grouped separately from the main group of compounds derived from the LOX pathway, among which their metabolic precursors could be found (Figure 3A). This distancing in the plot might be related to the aforementioned weak correlation found between these groups of compounds (Table 2), and to the inactivation of ADH during oil extraction [39]. The grouping of most of the esters in the fourth quadrant is evident, next to but separated from the main group of compounds synthesized through the LOX pathway. This might again be indicative of a disconnection with the mainstream LOX pathway. In this sense, there was no correlation between the LOX esters and their main 
precursors, the C6/LnA alcohols (Table 2), which might reflect the limitation of alcohol synthesis during VOO production rather than a true dampening of AAT activity. As expected, the $\mathrm{BC}$ compounds and terpenes grouped separately from each other and from the main group of compounds derived from the LOX pathway, reflecting their different metabolic origins.

PCA bi-plots of the progeny oils showed strong associations between the C6 and C5 compounds (Figure 3A) and a number of progeny genotypes present in the third quadrant (Figure 3B). Meanwhile, olive genotypes with a high content of esters and BC compounds derived from amino acid metabolism were situated in the fourth quadrant. Thus, it is possible to select genotypes from the progeny displaying a high level of a particular compound. However, while genotypes characterized with a high content of desirable compounds like C6/LnA aldehydes (6C-1 to 6C-4) could be identified in the plot, most of them were also rich in non-desirable compounds like pent-1-en-3-one (5C-1), considered to provide unpleasant sensations [13], or pent-1-en-3-ol (5C-4), which lies in the undesirable sector of the olive oil SSW [10].

A PCA was performed considering the major groups of volatile compounds in the progeny oils as variables in order to distinguish the genotypes that are especially rich in some of these (Figure 4). Genotypes giving rise to oils with high C6/LnA aldehyde contents are situated along the bisector of the first quadrant, while those producing oils with high C5/LnA content, which include some non-desirable compounds from a sensorial point of view, are located along the first factor axis. This distribution does not allow adequate selection of the genotypes whose oils have high C6/LnA aldehydes content as well as high concentrations of LOX esters or BC aldehydes., These latter groups are closely related (Figure 4A), such that genotypes producing oils rich in LOX esters commonly have high BC aldehyde contents, which might synergistically provide green-fruity odor notes as they are located in the green and ripe fruit sectors of the SSW [10]. Thus, it is possible to select genotypes from the progeny whose oils have a potential dominant green aroma, such as the genotypes UCI-55, UCI-125, UCI-40 and UCI-20, or with a potent ripe fruit aroma, such as UCI-74, UCI-13, UCI-135 and UCI-26.

As mentioned initially, the contribution of each volatile compound to the VOO aroma depends on its concentration and odor threshold. Only a few volatile compounds are present at levels indicating that they may contribute to the oil aroma $(\mathrm{OAV}>1)$ of all the progeny genotypes (Table 1). However, other volatile compounds contribute to just a given number of the oil genotypes. PCA was performed considering only those volatile compounds that might 
contribute to the aroma of the oil in more than 5\% of the genotypes as a variable (Figure 5). Most of these compounds were considered desirable for the aroma of VOO, except for hexan1-ol (6C-9), pent-1-en-3-one (5C-1), and pent-1-en-3-ol (5C-4), which provide unpleasant sensations according to literature [10, 13, 38]. Factors 1 and 2 explain a good amount of the data variation (41\%), and a vector distribution clearly distinguishes between desirable and undesirable areas in the genotype distribution plot when they are included as supplementary variables (Figure 5). The vector for desirable aroma characteristics runs almost along the bisector of the first quadrant, whereas the vector for undesirable aroma runs along the bisector of the fourth quadrant (Figure 5A). The progenitors are located on both sides of the first factor axis, although Arbequina is located in the sense of the desirable vector such that, in theory, Arbequina oil aroma would be more desirable than Picual oil aroma (Figure 5B). This appreciation might be related to the two-fold increase in C6/LnA compounds and the more than 50\% reduction of C5/LnA carbonyls in Arbequina oils compared to Picual oils. Genitors differences may also be observed in the results of the cluster analysis (Figure 6). When using as variables the volatile compounds that contribute to the aroma $(\mathrm{OAV}>1)$ of the oils, the genotypes from the progeny are distributed into two main groups. In each of these groups, both genitors occupy a quite central position, respectively. The distribution of vectors in Figure 5 allows genotypes such as UCI-41, UCI-36, UCI-39, UCI-68, UCI-133, or UCI-63 to be identified, which presumably give rise to oils with remarkable sensory properties. As displayed in Figure 6, most of these genotypes are included in the Picual group of the cluster analysis. This information could be of interest for breeding programs aimed at producing new cultivars with improved oil quality [21, 22].

In summary, this study shows that through a single cross of olive cultivars, it is possible to obtain a high degree of variability for the main components responsible for the aroma quality of VOO, which widely transgresses the variability in the progenitors. This finding suggests that in breeding programs, it might be more effective to consider a larger number of individuals within the same cross than using different crosses with fewer individuals, in close agreement with earlier studies [42,27]. The weak correlations found between most of the volatile components that might influence aroma suggest the possibility of obtaining new cultivars with a wide range of sensory profiles. The use of multivariate analysis allows particularly interesting genotypes to be identified in terms of the volatile compound composition and deduced organoleptic quality. Thus, the evaluation of the volatile profile at 
404

the initial stage of selection can serve to identify potential new olive cultivars in breeding programs that produce oils with improved sensory qualities.

\section{Acknowledgments}

This funding for this research came from the OLEAGEN project of Genoma España and the project AGL2011-24442 from the Programa Nacional de Recursos y Tecnologías Agroalimentarias, both financed by the Spanish Government. The plant materials evaluated here were obtained from the cooperative breeding program carried out at the University of Cordoba, and at the Institute of Agricultural and Fishery Research and Training, Spain.

\section{References}

[1] L. Lucas, A. Russell, R. Keast, Molecular mechanisms of inflammation. Antiinflammatory benefits of virgin olive oil and the phenolic compound oleocanthal, Curr. Pharm. Design 17 (2011) 754-768.

[2] R. Estruch, E. Ros, J. Salas-Salvadó, M.I. Covas, D. Corella, et al., Primary prevention of cardiovascular disease with a Mediterranean diet, N. Engl. J. Med. 368 (2013) 12791290.

[3] F. Visioli, E. Bernardini, Extra virgin olive oil's polyphenols: Biological activities. Curr. Pharm. Design 17 (2011) 786-804.

[4] V. Konstantinidou, M.I. Covas, D. Muñoz-Aguayo, O. Khymenets, R. de La Torre, et al, In vivo nutrigenomic effects of VOO polyphenols within the frame of the Mediterranean diet: a randomized trial, FASEB J. 24 (2010) 2546-2557.

[5] F. Pérez-Jiménez, , J. Ruano, P. Pérez-Martínez, F. López-Segura, J. López-Miranda, The influence of olive oil on human health: Not a question of fat alone, Mol. Nutr. Food Res. 51 (2007) 1199-1208. 
[6] T. Psaltopoulou, R.I. Kosti, D. Haidopoulos, M. Dimopoulos, D.B. Panagiotakos, . Olive oil intake is inversely related to cancer prevalence: a systematic review and a metaanalysis of 13800 patients and 23340 controls in 19 observational studies, Lipids Health Dis. 10 (2011) 127.

[7] M.T. Morales, R. Aparicio-Ruiz, R. Aparicio, Chromatographic methodologies: Compounds for olive oil odor issues, in: R Aparicio, J Harwood (Eds), Handbook of olive oil: Analysis and properties, Springer, New-York. 2013, pp. 261-309.

[8] P.Z. Bedoukian, The seven primary hexenols and their olfactory characteristics, J. Agric. Food Chem. 19 (1971) 1111-1114.

[9] A. Hatanaka, T. Kajiwara, H. Horino, K.I. Inokuchi, Odor-structure relationships in nhexanols and n-hexenales, Z. Naturforsch. 47 (1992) 183-189.

[10] R. Aparicio, M.T. Morales, Sensory wheels: A statistical technique for comparing QDA panels. Application to virgin olive oil, J. Sci. Food Agric. 67 (1995) 247-257.

[11] J.M. Olías, A.G. Pérez, J.J. Rios, C. Sanz, Aroma of virgin olive oil: biogenesis of the green odor notes, J. Agric. Food Chem. 41 (1993) 2368-2373.

[12] M.T. Morales, R. Aparicio, J.J. Ríos, Dynamic headspace gas chromatographic method for determining volatiles in virgin olive oil, J. Chromatogr. A 668 (1994) 455-462.

[13] F. Angerosa, R. Mostallino, C. Basti, R. Vito, Virgin olive oil odour notes: their relationships with the volatile compound from the lipoxigenase pathway and secoiridoid compounds, Food Chem. 68 (2000) 283-287.

[14] J.J. Salas, M. Williams, J.L. Harwood, J. Sánchez, Lipoxygenase activity in olive (Olea europaea) fruit, J. Amer. Oil Chem. Soc. 76 (1999) 1163-1169.

[15] J.J. Salas, J. Sánchez, Hydroperoxide lyase from olive (Olea europaea) fruits, Plant Sci. 143 (1999) 19-26.

[16] J.J. Salas, J. Sánchez, Alcohol dehydrogenases from olive (Olea europaea) fruit, Phytochemistry, 48 (1998) 35-40.

[17] J.J. Salas, Characterization of alcohol acyltransferase from olive fruit, J. Agric. Food Chem. 52 (2004) 3155-3158.

[18] H.W. Gardner, M.J. Grove, Y.P. Salch, Enzymic pathway to ethyl vinyl ketone and 2pentenal in soybean preparations, J. Agric. Food Chem., 44 (1996) 882-886.

[19] A.J. Fisher, H.D. Grimes, R. Fall, The biochemical origin of pentenol emissions from wounded leaves, Phytochemistry, 62 (2003) 159-163. 
[19] R. Aparicio, M.T. Morales, Sensory wheels: A statistical technique for comparing QDA panels. Application to virgin olive oil, J. Sci. Food Agric. 67 (1995) 247-257.

[20] S. Lavee, Aims, methods and advances in breeding of new olive (Olea europaea L.) cultivars, Acta Hortic. 286 (1989) 23-40.

[21] L. León, G. Beltrán, M.P. Aguilera, L. Rallo, D. Barranco, R. de la Rosa, Oil composition of advanced selections from an olive breeding program, Eur. J. Lipid Sci. Technol. 113 (2011) 870-875.

[22] I. Rjiba, N. Gazzah, S. Dabbou, M. Hammami, Evaluation of virgin olive oil minor compounds in progenies of controlled crosses, J. Food Biochem. 35 (2011) 1413-1423.

[23] F. Santos-Antunes, L. León, R. de la Rosa, J. Alvarado, A. Mohedo, I. Trujillo, L. Rallo, The length of the juvenile period in olive as influenced by vigor of the seedlings and the precocity of the parents, Hortscience 40 (2005) 1213-1215.

[24] M. El Riachy, F. Priego-Capote, L. Rallo, M.D. Luque de Castro, L. León, Phenolic profile of virgin olive oil from advanced breeding selections, Span. J. Agric. Res. 10 (2012) 443-453.

[25] J.M. Martínez, E. Muñoz, J. Alba, A. Lanzón, Report about the use of the 'Abencor' yields analyser, Grasas Aceites 26 (1975) 379-385.

[26] L. León L. Rallo, C. del Rio, L.M. Martín, Variability and early selection on the seedling stage for agronomic traits in progenies from olive crosses, Plant Breed. 123 (2004) 73-78.

[27] A.G. Pérez, L. León, M. Pascual, C. Romero-Segura, A. Sánchez-Ortiz, R. de la Rosa, C. Sanz, Variability of virgin olive oil phenolic compounds in a segregating progeny from a single cross in Olea europaea L. and sensory and nutritional quality implications, PLOS ONE 9 (2014) e92898.

[28] A. Sánchez-Ortiz, A.G. Pérez, C. Sanz, Cultivar differences on nonesterified polyunsaturated fatty acid as a limiting factor for biogenesis of virgin olive oil aroma, $\mathrm{J}$. Agric. Food Chem. 55 (2007) 7869-7873.

[29] A. Sánchez-Ortiz, C. Romero-Segura, C. Sanz, A.G. Pérez, Synthesis of volatile compounds of virgin olive oil is limited by the lipoxygenase activity load during the oil extraction process, J. Agric. Food Chem. 60 (2012) 812-822.

[30] A. Sánchez-Ortiz, A.G. Pérez, C. Sanz, Synthesis of aroma compounds of virgin olive oil: Significance of the cleavage of polyunsaturated fatty acid hydroperoxides during the oil extraction process, Food Res. Int. 54 (2013) 1972-1978. 
[31] F. Angerosa, Influence of volatile compounds on virgin olive oil quality evaluated by analytical approaches and sensor panels, Eur. J. Lipid Technol. 104 (2002) 639-660.

[32] M.T. Morales, G. Luna, R. Aparicio, Comparative study of virgin olive oil sensory defects, Food Chem. 91 (2005) 293-301.

[33] G. Luna, M.T. Morales, R. Aparicio, Characterisation of 39 varietal virgin olive oils by their volatile composition, Food Chem. 98 (2006) 243-252.

[34] F. Angerosa, L. Camera, N. d'Alessandro, G. Mellerio, Characterization of seven new hydrocarbon compounds present in the aroma of virgin olive oils, J. Agric. Food Chem. 46 (1998) 648-653.

[35] C.D. Evans, H.A. Moser, G.R. List, Odor and flavor responses to additives in edible oils, J. Amer. Oil Chem. Soc. 48 (1971) 495-498.

[36] R. Aparicio, M.T. Morales, M.V. Alonso, Relationship between volatile compounds and sensory attributes of olive oils by sensory wheel, JAOCS 73 (1996) 1253-1264.

[37] F. Angerosa, R. Mostallino, C. Basti, R. Vito, Virgin olive oil odour notes: their relationship with volatile compounds from the lipoxygenase pathway and secoiriodoid compounds, Food Chem. 68 (2000) 283-287.

[38] F. Angerosa, B. Lanza, V. Marsilio, Biogenesis of "fusty" defect in virgin olive oils, Grasas Aceites 47 (1996) 142-150.

[39] A. Sánchez-Ortiz, C. Romero-Segura, V.E. Gazda, I.A. Graham, C. Sanz, A.G. Pérez, Factors limiting the synthesis of virgin olive oil volatile esters, J. Agric. Food Chem. 60 (2012) 1300-1307.

[40] M. El Riachy, F. Priego-Capote, L. León, M.D. Luque de Castro, L. Rallo, Virgin olive oil phenolic profile and variability in progenies from olive crosses, J. Sci. Food Agric. 92 (2012) 2524-2533.

[41] C.M. Kalua, D.R. Bedgood, A.G. Bishop, P.D. Prenzler, Changes in volatile and phenolic compounds with malaxation time and temperature during virgin olive oil production, J. Agric. Food Chem. 54 (2006) 7641-7651.

[42] M. El Riachy, F. Priego-Capote, L. Rallo, M.D. Luque de Castro, L. León, Phenolic composition of virgin olive oils from cross breeding segregating populations, Eur. J. Lipid Sci. Technol. 114 (2012) 542-551.

[43] W. Grosch, Determination of potent odorants in foods by aroma extract dilution analysis (AEDA) and calculation of odour activity values (OAVs), Flavour Fragr. J. 9 (1994) $147-158$. 
[44] J. Reiners, W. Grosch, Odorants of virgin olive oils with different flavor profiles, J. Agric. Food Chem. 46 (1998) 2754-2763.

[45] R. Aparicio, S.M. Rocha, I. Delgadillo, M.T. Morales, Detection of rancid defect in virgin olive oil by the electronic nose, J. Agric. Food Chem. 48 (2000) 853-860.

[46] R. Aparicio, G. Luna, Characterisation of monovarietal virgin olive oils, Eur. Lipid Sci. Technol. 10 (2002) 614-627.

[47] R.A. Flath, R.R. Forrey, D.G. Guadagni, Aroma components of olive oil, J. Agric. Food Chem. 21 (1973) 948-952.

[48] D. Tura, O. Failla, D. Bassi, S. Pedò, A. Serraiocco, Cultivar influe ce of virgin olive (Olea europea L.) oil flavor based on aromatic compounds and sensorial profile, Sci. Hortic. 118 (2008) 139-148.

[49] M.T. Morales, M.V. Alonso, J.J. Rios, R. Aparicio, Virgin olive oil aroma: relationship between volatile compounds and sensory attributes by chemometrics, J. Agric. Food Chem. 43 (1995) 2925-2931.

[50] M.T. Morales, R. Aparicio, Effect of extraction conditions on sensory quality of virgin olive oil, J. Am. Oil Chem. Soc. 76 (1999) 295-300.

[51] H. Guth, W. Grosch, Quantitation of potent odorants of virgin olive oil by stable-isotope dilution assays, JAOCS 70 (1993) 513-518.

[52] E. Fedeli, Caratteristiche organolettiche dell'olio di oliva, Riv. Ital. Sost. Grasse 54 (1977) 202-205. 


\section{Figure captions}

Figure 1. Content (ng/g oil) of the main groups of volatile compounds in the oils from the Picual x Arbequina progeny. The parental oils are indicated in the chart with arrows.

Figure 2. Range and distribution of the different classes of volatile compounds (ng/g oil) within the main groups in the oils from the Picual x Arbequina progeny. The squares in the interior of the boxes are the median values. The height of a box is equal to the interquartile distance, indicating the distribution for $50 \%$ of the data. The outliers (open dots) and extreme data (open triangles) are indicated outside the whiskers (the lines extending from the top and bottom of the box).

Figure 3. Bi-plot of the main volatile compounds in the oils from the Picual $x$ Arbequina progeny. Factors 1 and 2 explain $37.20 \%$ of the data variation. A: vector distribution of the volatile compounds grouped according to their metabolic origin. B: distribution of the genotypes from the progeny.

Figure 4. Bi-plot of the main groups of volatile compounds in the oils from the Picual $x$ Arbequina progeny. Factors 1 and 2 explain $38.32 \%$ of the data variation. A: vector distribution of the groups of volatile compounds (solid circles). B: distribution of the genotypes from the progeny, including the progenitors.

Figure 5. Bi-plot of selected volatile compounds that contribute to the aroma (OAV > 1) of the oils from the Picual x Arbequina progeny. Factors 1 and 2 explain $41 \%$ of the data variation. A: vector distribution of the volatile compounds (solid circles) and qualitative descriptors calculated from the corresponding compounds (open circles). B: distribution of the genotypes from the progeny, including the progenitors.

Figure 6. Cluster analysis of the genotypes of the Picual x Arbequina progeny using as variables the volatile compounds that contribute to the aroma $(\mathrm{OAV}>1)$ of the oils. The position of the genitors (Arbequina and Picual) is marked as well as the genotypes presumably producing oils with remarkable sensory properties $(*)$; from top to bottom: UCI-68, UCI-133, UCI-36, UCI-41, UCI-63, and UCI-39. 


\section{Supporting Information captions}

Table S1. Identification of virgin olive oil volatile compounds by means of SPME-GCMS analysis.

Figure S1. GC-MS analysis of the volatile fraction of virgin olive oil from cultivar Picual. Peak numbers are compounds listed in Table S1.

Figure S2. Principal component analysis distribution of the genotypes from the Picual $x$ Arbequina progeny taking all the volatile compounds as variables (A) and those most important from a sensorial point of view $(\mathrm{OAV}>1$ : B). The symbols for the genotypes have different colors according to the crop year. Prediction ellipses are displayed for each crop year $($ coefficient $=0.95)$. 